\title{
Regulation of Axonal Extension and Growth Cone Motility by Calmodulin-Dependent Protein Kinase I
}

\author{
Gary A. Wayman, ${ }^{1,2 *}$ Stefanie Kaech, ${ }^{3 *}$ Wilmon F. Grant, ${ }^{1}$ Monika Davare, ${ }^{1}$ Soren Impey, ${ }^{1}$ Hiroshi Tokumitsu, ${ }^{4}$ \\ Naohito Nozaki, ${ }^{5}$ Gary Banker, ${ }^{3}$ and Thomas R. Soderling ${ }^{1}$ \\ ${ }^{1}$ Vollum Institute, ${ }^{2}$ Department of Cell and Developmental Biology, and ${ }^{3}$ Center for Research on Occupational and Environmental Toxicology, Oregon \\ Health and Science University, Portland, Oregon 97239-3011, ${ }^{4}$ Department of Signal Transduction Sciences, Faculty of Medicine, Kagawa University, \\ Kagawa 761-0793, Japan, and ${ }^{5}$ Kanagawa Dental College, Yokosuka, Kanagawa 238-8580, Japan
}

Calcium and calmodulin $(\mathrm{CaM})$ are important signaling molecules that regulate axonal or dendritic extension and branching. The $\mathrm{Ca}^{2+}$-dependent stimulation of neurite elongation has generally been assumed to be mediated by CaM-kinase II (CaMKII), although other members of the CaMK family are highly expressed in developing neurons. We have examined this assumption using a combination of dominant-negative CaMKs (dnCaMKs) and other specific CaMK inhibitors. Here we report that inhibition of cytosolic CaMKI, but not CaMKII or nuclear CaMKIV, dramatically decreases axonal outgrowth and branching in cultured neonatal hippocampal and postnatal cerebellar granule neurons. CaMKI is found throughout the cell cytosol, including the growth cone. Growth cones of neurons expressing dnCaMI or dnCaMKK, the upstream activator of CaMKI, exhibit collapsed morphology with a prominent reduction in lamellipodia. Live-cell imaging confirms that these morphological changes are associated with a dramatic decrease in growth cone motility. Treatment of neurons with 1,8-naphthoylene benzimidazole-3-carboxylic acid (ST0-609), an inhibitor of CaMKK, causes a similar change in morphology and reduction in growth cone motility, and this inhibition can be rescued by transfection with an ST0-609-insensitive mutant of CaMKK or by transfection with constitutively active CaMKI. These results identify CaMKI as a positive transducer of growth cone motility and axon outgrowth and provide a new physiological role for the CaMKK-CaMKI pathway.

Key words: calcium; Ca; calmodulin; growth cone; hippocampus; kinase; neurite

\section{Introduction}

Neurite extension and growth cone dynamics show complex responses that depend on the localization, concentration, and temporal dynamics of the $\mathrm{Ca}^{2+}$ signal (Kater and Mills, 1991; Petersen and Cancela, 2000). Many stimulatory effects of $\mathrm{Ca}^{2+}$ on neurite outgrowth are blocked by the compound 1-[N,O-bis(5isoquinolinesulfonyl)- $N$-methyl-L-tyrosyl]-4-phenylpiperazine (KN-62) (Zheng et al., 1994; Solem et al., 1995; Williams et al., 1995; Kuhn et al., 1998; Vaillant et al., 2002). Because KN-62 was originally thought to be a specific calmodulin kinase II (CaMKII) inhibitor (Tokumitsu et al., 1990), it was assumed that CaMKII mediated these stimulatory $\mathrm{Ca}^{2+}$ effects; however, $\mathrm{KN}-62$ is now recognized to also inhibit CaMKIV and CaMKI (Mochizuki et al., 1993; Enslen et al., 1994). Furthermore, the effects of CaMKII expression on neurite outgrowth are unclear. Expression of active CaMKII enhances neurite extension in neuroblastoma cells (Goshima et al., 1993; Sogawa et al., 2000) but suppresses it in

\footnotetext{
Received July 10, 2003; revised March 1, 2004; accepted March 2, 2004.

This work was supported by National Institutes of Health Grants DK44239 (T.R.S.), NS27037 (T.R.S.), and NS17112 (G.B.). Antibodies to CaMKI were kindly provided by Dr. Angus Nairn (Yale University) and Dr. Hitoshi Fujisawa (Shiga Medical Center Research Institute). Thanks also to Barbara Smoody for preparing hippocampal cultures.

${ }^{*}$ G.A.W. and S.K. contributed equally to this work.

Correspondence should be addressed to Thomas R. Soderling, Vollum Institute, Oregon Health and Science University, 3181 SW Sam Jackson Park Road, Portland, OR 97239. E-mail: soderlit@ohsu.edu.

D0I:10.1523/JNEUROSCI.3294-03.2004

Copyright $\odot 2004$ Society for Neuroscience $\quad$ 0270-6474/04/243786-09\$15.00/0
}

PC12 cells (Tashima et al., 1996) and Xenopus retinotectal neurons ( $\mathrm{Wu}$ and Cline, 1998). Recent work indicates that $\beta$, but not $\alpha$, CaMKII can have stimulatory effects on dendritic arborization (Fink et al., 2003). Hence, it is important to reexamine the question of which CaMKs are involved in regulating axonal extension and growth cone motility to elucidate the relevant signaling pathways.

Other CaMKs abundant in neurons include members of the CaMK cascade: CaMKI, CaMKIV, and CaMKK (for review, see Soderling, 1999; Means, 2000). CaMKK, which is present in both the cytoplasm ( $\alpha$ isoform) and nucleus ( $\beta$ isoform) (Sakagami et al., 2000; Nakamura et al., 2001), phosphorylates and activates CaMKI and CaMKIV in response to elevations of intracellular $\mathrm{Ca}^{2+}$. CaMKK is present in neurons in most brain regions, but its developmental pattern of expression is not known. CaMKIV is restricted primarily to the nucleus (Jensen et al., 1991), where it phosphorylates several transcription factors such as cAMP response element-binding protein (CREB) and its coactivator CREB binding protein (Impey et al., 2002). Various isoforms of CaMKIV (Sakagami et al., 1992) exhibit high expression throughout development in hippocampal pyramidal and cerebellar granule neurons. In neurons, CaMKI is predominantly cytoplasmic (Picciotto et al., 1995); however, a splice variant of the $\beta$ isoform is nuclear (Rina et al., 2001), and the $\gamma$ isoform can be associated with the Golgi and plasma membranes (TakemotoKimura et al., 2003). Developmentally, mRNA for CaMKI iso- 
forms in rat brain is present at embryonic day (E) 15, increases at E18, and peaks at postnatal day (P) 1-7 (Sawamura et al., 1996; Loseth et al., 2000). CaMKI is present in numerous brain regions, including hippocampal pyramidal and cerebellar granule neurons, cell types that are examined in this report. Although several in vitro substrates for CaMKI are known (Nairn and Greengard, 1987; Picciotto et al., 1992; Lee et al., 1994), in vivo substrates are unknown, and therefore physiological roles for CaMKI have been essentially unexplored.

Using a combination of dominant-negative ( $\mathrm{dn}$ ) kinases and other specific kinase inhibitors, we find that CaMKI regulates axonal extension and growth cone motility in cultured hippocampal and cerebellar nerve cells. These results show that CaMKI plays a previously unsuspected role in the regulation of actin-based motility in developing neurons.

\section{Materials and Methods}

Cell culture. High-density hippocampal neurons $\left(2 \times 10^{5}\right.$ cells per square centimeter) were cultured according to the procedure of Brewer (1997) from P0-2 Sprague Dawley rats on plates coated with poly-D-lysine (Sigma, St. Louis, MO; molecular weight $>300,000$ ). The hippocampal neurons were maintained in Neurobasal A media (Invitrogen, Gaithersburg, MD) supplemented with B27 (Invitrogen) and $0.5 \mathrm{~mm}$ L-glutamine with $5 \mu \mathrm{M}$ cytosine $\beta$-D-arabinofuranoside (AraC) added at day 2 in vitro (2 DIV). Low-density hippocampal neuronal cell cultures $\left(\sim 10^{4}\right.$ neurons per square centimeter) were prepared from E18 rats as described previously (Goslin and Banker, 1998). Cerebellar granule cell cultures were prepared from P6 8 Sprague Dawley rats using papain dissociation (Worthington Biochemical, Freehold, NJ). The cerebellar granule cells were plated $\left(2-4 \times 10^{5}\right.$ cells per square centimeter $)$ on borosilicate glass coverslips coated with poly D-lysine and grown in Neurobasal A media supplemented with $2 \times$ B27, $4 \%$ FBS, $0.5 \mathrm{~mm}$ L-glutamine, $25 \mathrm{~mm} \mathrm{KCl}$, and $5 \mu \mathrm{M}$ AraC. None of the CaMK transfections or 1,8-naphthoylene benzimidazole-3-carboxylic acid (STO-609) treatment described in this work had noticeable effects on apoptosis as assessed by condensed nuclei using Hoechst staining.

Vector constructs. All of the CaMK constructs used in this study were the $\alpha$ isoforms. The expression vectors for dnCaMKK and dnAkt (Yano et al., 1998), dnCaMKIV (Gringhuis et al., 1997), and CaMKIIN (Chang et al., 2001) have been described previously. The dnCaMKI was generated by a series of point mutations: K49E, T177A, 286IHQS to 286EDDD, and $307 \mathrm{~F}$ to A. The K49 (catalytic lysine) to E mutation disrupts catalytic activity. The T177 (activation loop threonine) to A mutation prevents CaMKK phosphorylation of the activation loop of CaMKI. Mutations 286IHQS to EDDD and 307F to A (Matsushita and Nairn, 1998) disrupt the autoinhibitory domain, thereby mimicking the effect of $\mathrm{Ca}^{2+}-$ CaM-binding. The constitutively active CaMKI (caCaMKI) mutant was identical to dnCaMKI but without the K49E and T177A mutations. The enhanced green fluorescent protein (EGFP)-tagged wild-type (wt) CaMKI, dnCaMKI, and wtCaMKK were constructed using the plasmid EGFP (pEGFP) vector (Clontech, Cambridge, UK). Nuclearrestricted dnCaMKIV (dnCaMKIVnuc) was constructed using the pEGFPnuc vector (Clontech).

Transfections. Primary hippocampal neurons and cerebellar granule neurons were transfected with LipofectAMINE 2000 (Invitrogen) according to the manufacturer's protocols. In each culture type, we optimized DNA amounts, transfection reagent amounts, and transfection durations to yield minimal toxicity and maximal transfection efficiency.

Microscopy and quantification of neurite outgrowth and growth cone motility. After transfection, cells were allowed to grow for varying times ( $8 \mathrm{hr}$ to $2 \mathrm{~d}$ ), fixed with $4 \%$ paraformaldehyde, $4 \%$ sucrose, $50 \mathrm{~mm}$ HEPES, PBS, pH 7.5, for 10-15 min at room temperature, and then permeabilized with $0.25 \%$ Triton X-100 in PBS. Filamentous actin was visualized using Texas Red-labeled phalloidin (Sigma). Indirect immunofluorescence with anti-tubulin antibody (Ab) clone 6-11B-1 (Sigma) was used to visualize microtubules. Cells were viewed on a Zeiss Axiophot or a Leica DM RXA microscope using high numerical aperture lenses, and the images were recorded with a cooled CCD camera. Total process lengths were measured using MetaMorph software (Universal Imaging, West Chester, PA). Axonal and dendritic processes were identified by their morphological characteristics (Goslin and Banker, 1998). High-resolution confocal images were acquired using an Olympus FluoView FV300 confocal laser scanning microscope. For live imaging, cells were maintained in a sealed, heated chamber (Warner Instruments) in balanced salt solution supplemented with $0.6 \%$ glucose. Care was taken to attenuate excitation light and shutter light exposure to prevent growth cone collapse caused by phototoxicity. Images were captured every $5 \mathrm{sec}$ over a time period of $10 \mathrm{~min}$. For analysis of motility, outlines of growth cones drawn on individual frames were overlaid in Adobe Photoshop, and the area occupied by the growth cone in all frames (black) was contrasted with the area visited by the growth cone in only some of the frames (depicted in white).

\section{Results}

\section{CaMKII does not stimulate axon outgrowth}

To identify the CaMKs that mediate the effects of $\mathrm{Ca}^{2+}$ on axon development, we focused on two well characterized cell culture models, neonatal hippocampal neurons grown under standard conditions and postnatal cerebellar neurons grown in slightly depolarizing conditions, that are routinely used to prevent apoptosis. We first explored the possible involvement of CaMKII, a multifunctional kinase known to regulate several neuronal functions (for review, see Soderling et al., 2001; Lisman et al., 2002), because it had been implicated in previous studies (Zheng et al., 1994; Solem et al., 1995; Williams et al., 1995; Kuhn et al., 1998; Vaillant et al., 2002). Rather than using KN-62, which inhibits various CaMK family members, we overexpressed CaMKIIN, an endogenous protein inhibitor of CaMKII (Chang et al., 1998, 2001). CaMKIIN inhibits both the $\alpha$ and $\beta$ brain isoforms of CaMKII $\left(\mathrm{IC}_{50} \sim 50 \mathrm{nM}\right.$ ), whereas concentrations up to $10 \mu \mathrm{M}$ show little inhibition of CaMKK, CaMKI, CaMKIV, protein kinase A (PKA), or PKC. CaMKIIN inhibits the CaM-dependent activity of CaMKII and, unlike KN-62, also blocks constitutive CaMKII activity because of its activation through autophosphorylation of Thr286.

To determine whether overexpression of CaMKIIN alters axon outgrowth, we transfected hippocampal or cerebellar granule neurons on day 2 in culture with CaMKIIN and EGFP (to label transfected cells) or with EGFP alone as a control. Cultures were fixed on day 4 , and neurite length and branching were analyzed in fluorescent cells. At this stage of development both axons and immature dendrites are growing rapidly, with the axon accounting for $\sim 85 \%$ of total neurite length. Overexpression of CaMKIIN effectively blocked KCl-induced activation of CaMKII (i.e., its autophosphorylation on Thr286), but did not reduce total neurite length, the average number of neurites projecting from the cell body, or the number of neurite terminals, a measure of neurite branching (Fig. 1). In fact, CaMKIIN had a slight stimulatory effect in cerebellar granule neurons (numbers of processes and terminals), suggesting that CaMKII may be inhibitory.

\section{CaMKK and CaMKI stimulate axon extension}

Because these data indicate that CaMKII does not play a stimulatory role in axon extension in hippocampal or cerebellar neurons, we next investigated other members of the CaMK family using "kinase dead" dominant-negative constructs. Neurons (2 DIV) were transfected with vectors expressing dnCaMKs (see Materials and Methods for details of constructs) fused to EGFP, cultured for an additional $2 \mathrm{~d}$, and fixed, and fluorescent cells were analyzed. Hippocampal neurons expressing EGFP alone had the typical morphology of young, stage 4 cells (Dotti et al., 
A

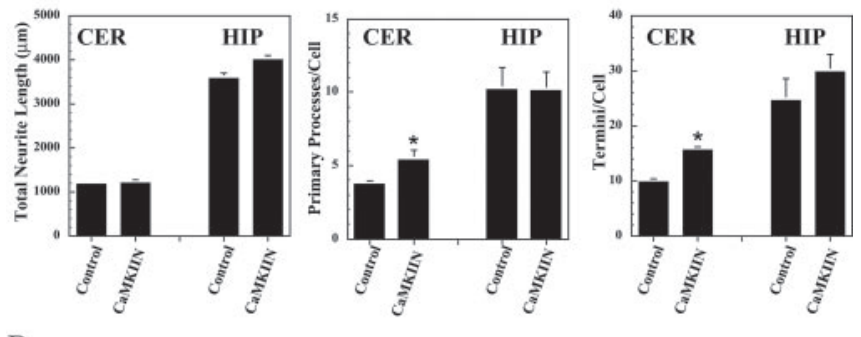

B

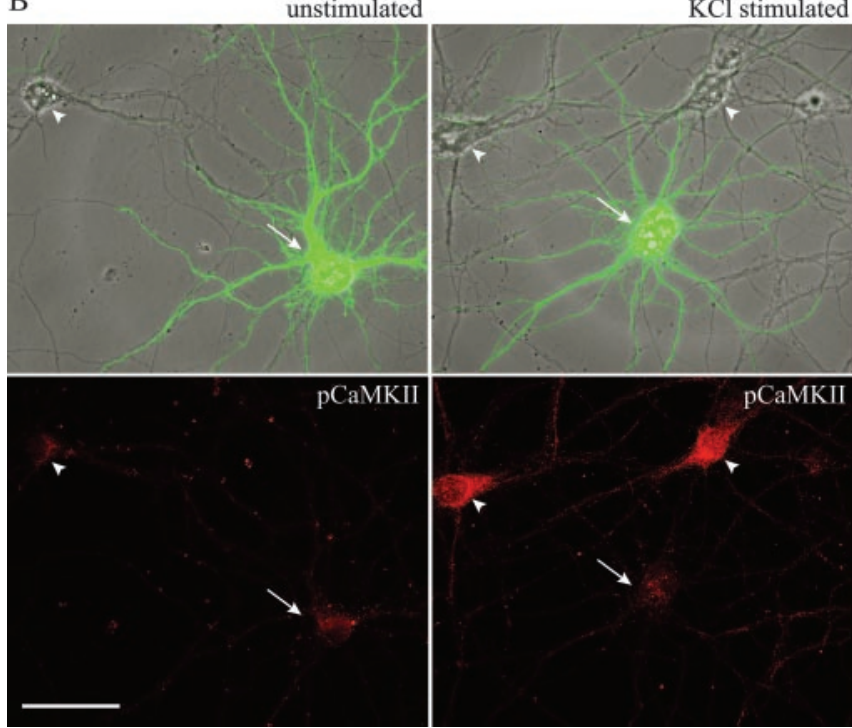

Figure 1. Neurite growth in hippocampal and cerebellar granule cells is not suppressed by inhibition of CaMKII. A, High-density cultures of rat hippocampal (HIP) and cerebellar granule cell neurons (CER) were transfected on day 2 with plasmid encoding soluble EGFP alone (control) or in combination with the specific CaMKII inhibitor, CaMKIIN. Neurons were cultured an additional $2 \mathrm{~d}$ and then fixed and imaged. Results show total neurite length, number of primary neurites, and the number of neurite terminals (reflecting neurite number and branching), based on the analysis of 30 cells in each condition (means $\pm S D$ ). ${ }^{*} p$ values of $<0.01$ ( $t$ test). $B$, CaMKIIN inhibits CaMKII activity in hippocampal neurons. Low-density hippocampal cultures were transfected on day 5 with plasmid encoding EGFP-CaMKIIN. The neurons were cultured an additional $9 \mathrm{~d}$ and then stained with phospho-dependent Ab for Thr286 in CaMKII (pCaMKII), either with (right panels) or without (left panels) previous stimulation with $90 \mathrm{~mm} \mathrm{KCl} \mathrm{(5} \mathrm{min).}$ After $\mathrm{KCl}$ stimulation, nontransfected neurons (arrowheads) show enhanced staining for activated CaMKII (autophosphorylated at Thr286) throughout their cell bodies and processes (bottom panel). EGFP-CaMKIIN-expressing neurons (arrows) show no increase in anti-phosphoCaMKII staining over levels seen in unstimulated cells, demonstrating that overexpression of CaMKIIN inhibited activation of endogenous CaMKII. Scale bars: $A, 10 \mu \mathrm{m} ; B, 50 \mu \mathrm{m}$.

1988), with one long branched axon and several shorter dendrites (Fig. 2 A, Control). Total neurite length averaged $3000 \mu \mathrm{m}$ (Fig. $2 B)$. Expression of dnCaMKI, dnCaMKIV, or dnCaMKK suppressed neurite length by $\sim 70 \%$ and also significantly reduced the numbers of primary processes and termini per cell (Fig. $2 \mathrm{~A}-$ $C)$. A similar dramatic inhibition of neurite extension, number of primary processes, and number of termini was observed in cerebellar granule neurons expressing these dominant-negative constructs (Fig. 3). Growth of both axons and immature dendrites was inhibited in both cell types.

It is expected that dnCaMKK would have effects similar to dnCaMKI or dnCaMKIV because CaMKK is the upstream activator for both of these kinases. The dnCaMKI and dnCaMKIV probably act by sequestering the upstream CaMKK. If so, expression of dnCaMKI should block CaMKK-mediated phosphorylation of Thr177 in endogenous CaMKI. Using a phospho-specific
Ab to phospho-Thr177 in CaMKI (supplemental Fig. S1 A, available at www.jneurosci.org), we show that transfection of NG108 neuroblastoma cells with dnCaMKI strongly suppresses activation of endogenous CaMKI (supplemental Fig. S1 B). NG108 cells were used because the transfection efficiency is $\sim 80 \%$ compared with $1-3 \%$ efficiency in hippocampal neurons. However, it was surprising that dnCaMKIV was also inhibitory to neurite outgrowth (Fig. 2), because the subcellular distributions of CaMKI and CaMKIV are very different in neurons. It is well established that CaMKIV is mostly nuclear (Jensen et al., 1991), whereas CaMKI is predominantly cytoplasmic (Picciotto et al., 1995). Immunostaining of cultured hippocampal neurons confirmed that endogenous CaMKI was cytosolic, including expression throughout the axonal and dendritic arbors (Fig. 4A). Because overexpressed proteins can exhibit anomalous subcellular distribution, we examined this issue. As expected, the $\alpha$ isoforms of EGFP-tagged wtCaMKI (Fig. $4 B$ ) and wtCaMKK (Fig. 4C), as well as EGFP-tagged dnCaMKI and dnCaMKK (data not shown), were present in the cytoplasm of transfected neurons and were essentially excluded from the nucleus. In contrast, expressed EGFP-tagged dnCaMKIV was not restricted to the nucleus but showed strong cytoplasmic expression that extended out into the dendrites and axon (supplemental Fig S1 $A$, top panels). To direct localization of dnCaMKIV to the same subcellular locale as endogenous CaMKIV, we added a nuclear localization signal (nuc) to EGFP-dnCaMKIV. As predicted, EGFPtagged dnCaMKIVnuc was restricted to the nucleus (supplemental Fig S1A, bottom panels). Unlike mislocalized dnCaMKIV, dnCaMKIVnuc had little effect on neurite growth and branching in either hippocampal (Fig. 2) or cerebellar (Fig. 3) neurons, but it was highly effective at blocking CREB-dependent gene transcription (supplemental Fig. S1 B). These results demonstrate that the nuclear effects of dnCaMKIV are not involved in regulating axon outgrowth at this stage of development and that the observed inhibitory effect of dnCaMKIV was caused by its abnormal cytosolic localization.

Because the activation site Ser308 in the protein kinase Aktprotein kinase B PKB is also a substrate for CaMKK (Yano et al., 1998), albeit relatively poor compared with CaMKI or CaMKIV, we also determined the effects of dnAkt. Transfected EGFPdnAkt had no significant effect on total neurite length (supplemental Fig. S2). We have previously used this dnAkt to inhibit Akt in NG108 cells (Yano et al., 1998). Taken together, these data indicate that CaMKK and CaMKI, but not Akt or nuclear CaMKIV, regulate axon extension under our conditions.

\section{Role for CaMKI in growth cones}

We were especially interested in the role of CaMKs in the growth cone, which functions both as a sensor of chemical cues (e.g., $\mathrm{Ca}^{2+}$ signaling) and as a mechanical transducer (via actin reorganization) to promote axon outgrowth (Gomez and Spitzer, 2000). CaMKI, localized with two separate antibodies, extended throughout the neurites and was present in axonal growth cones (Fig. 4A). To further explore the subcellular localization of these kinases, we analyzed the distribution of EGFP-tagged kinases in cultures of hippocampal neurons prepared at low density so that the morphology of individual neurons could be visualized more easily. Wild-type EGFP-tagged CaMKK and CaMKI were present throughout the axon and dendrites, extending into the axonal growth cone (Fig. 4B, C). Similar results were obtained with myctagged CaMKK and CaMKI (data not shown), excluding the effects of the EGFP tag on localization of these kinases.

Because CaMKK and its downstream substrate CaMKI are present in axonal growth cones, we examined the effects of inhibiting CaMKI on growth cone morphology. Low-density 
A

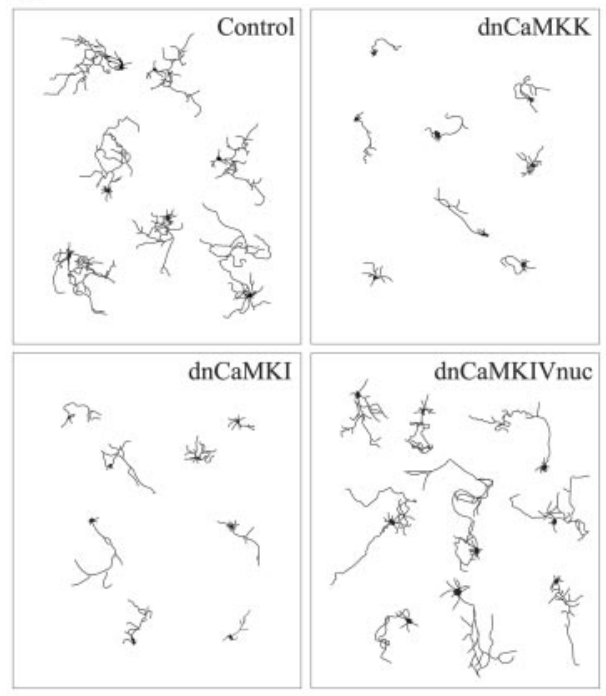

B

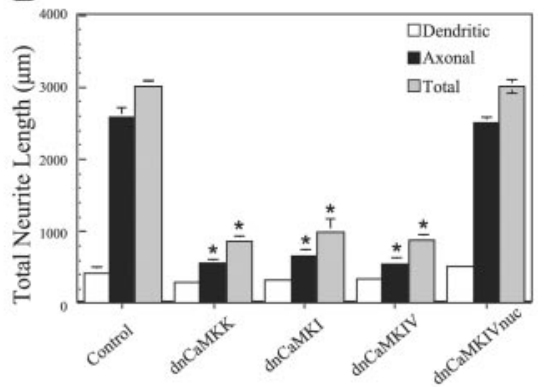

C

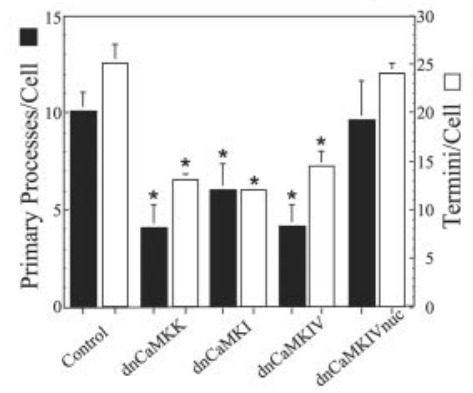

Figure 2. Hippocampal neurite growth is suppressed by inhibition of the CaMKK-CaMKI pathway but not by inhibition of nuclear CaMKIV. High-density cultures of rat hippocampal neurons were transfected on day 2 with plasmid encoding soluble EGFP alone (control) or in combination with dnCaKK, dnCaMKI, dnCaMKIV, or dnCaMKIVnuc. Neurons were cultured an additional $2 \mathrm{~d}$ and then fixed and imaged. Camera lucida drawings $(A)$ of control and CaMK-expressing neurons are shown. Quantification of the effects of inhibiting CaMKs K, I, or IV are shown in $B$ and $C$. Axons and dendrites were identified by morphology, and neurite growth was quantified as in Figure 1 ( 30 cells per condition). ${ }^{*} p$ values of $<0.01$ ( $t$ test).

A

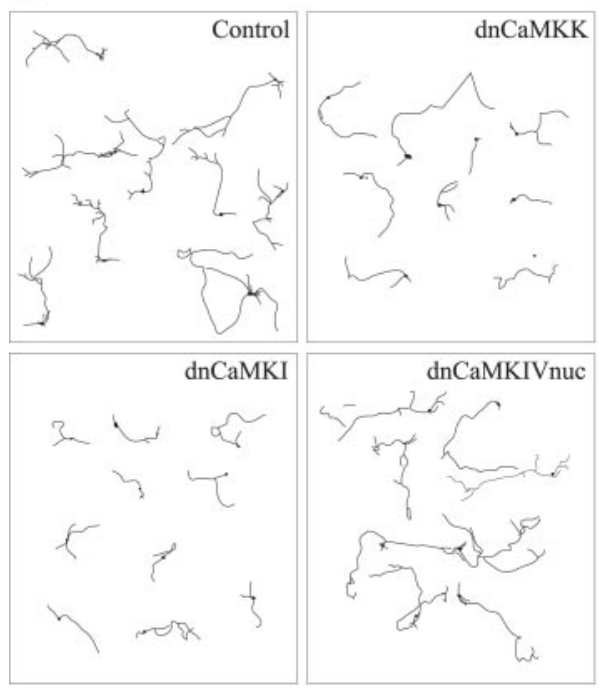

B

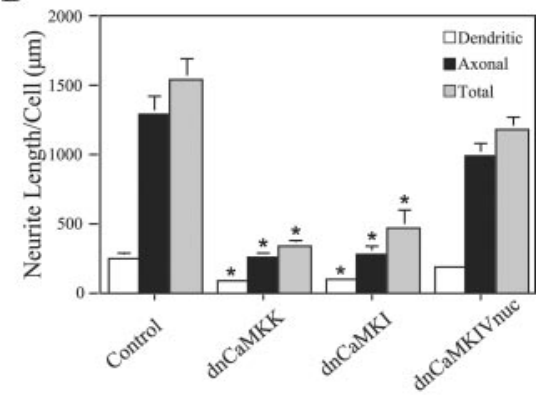

$\mathrm{C}$

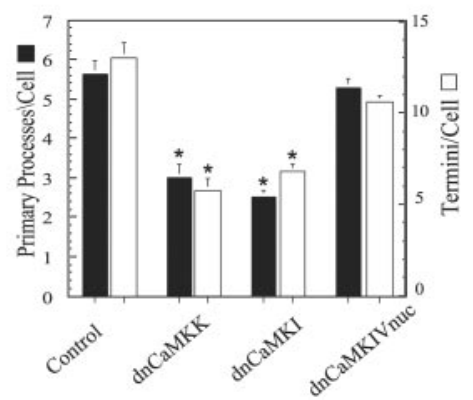

Figure 3. Cerebellar granule cell neurite growth is suppressed by inhibition of the CaMKK-CaMKI pathway. High-density cultures of rat cerebellar granule cell neurons were transfected on day 2 with plasmid encoding soluble EGFP alone (control) or in combination with dnCaKK, dnCaMKI, or dnCaMKIVnuc. Neurons were cultured an additional $2 \mathrm{~d}$ and then fixed and imaged. Camera lucida drawings $(A)$ of control and CaMK-expressing neurons are shown. Quantification of the effects of inhibiting CaMKs $\mathrm{K}, \mathrm{I}$, or IV are shown in $B$ and $C$. Axons and dendrites were identified by morphology, and neurite growth was quantified as in Figure 1 (30 cells per condition). ${ }^{*} p$ values of $<0.01$ ( $t$ test).

hippocampal cultures were transfected with EGFP-tagged dnCaMKI, and $8 \mathrm{hr}$ later neurons were fixed and stained for both filamentous actin and microtubules. Axonal growth cones of neurons expressing dnCaMKI were dramatically different from those of cells expressing EGFP alone or EGFP-wtCaMKI (Fig. 5). Their growth cones appeared "collapsed," with reduced lamellipodial area and a strong reduction in the number of filopodia (Fig. 5, middle panel, left side). Microtubules in the axon shafts appeared essentially unaffected by dnCaMKI. These data suggest that CaMKK and CaMKI may support axon extension by regulating the growth cone cytoskeleton.

\section{CaMKI regulates growth cone motility}

To examine the role of the CaMKKCaMKI pathway on axonal growth cone dynamics, low-density hippocampal cultures were transfected with either EGFP, EGFP-wtCaMKI, or EGFP-dnCaMKI, and remodeling of growth cones was followed by live-cell imaging. Images of EGFP fluorescence were obtained at $5 \mathrm{sec}$ intervals for a period of $10 \mathrm{~min}$. Movies illustrating growth cone movements are provided as supplemental material (available at www. jneurosci.org), and selected frames from these movies are shown in Figure 6. Control neurons exhibited prominent lamellipodial activity (extension and retraction of veils between filopodia) as well as filopodial motility (supplemental movie 1) (Fig. $6 A)$. In the peripheral region of the growth cone, a continuous, rearward flow of fluorescence was seen, probably corresponding to the retrograde flow of actin and plasma membrane observed by others (Lin and Forscher, 1995). Expression of wtCaMKI slightly enhanced growth cone size and motility (Figs. 5, 6B) (supplemental movie 2). In contrast, expression of dnCaMKI markedly reduced axonal growth cone motility (supplemental movies 3 and 4) (Fig. 6C). Lamellipodial extension was blocked, and the rearward flow of fluorescence was inhibited. The number of filopodia was greatly reduced, and the few filopodia that remained were frozen. At $8 \mathrm{hr}$ after transfection of dnCaMKI, motility was blocked in some cells and reduced in others (Fig. $6 C$, top panel) (supplemental movie 3). By 20-24 hr, motility was effectively blocked in all transfected cells (Fig. 6C, bottom panel) (supplemental movie 4). Images summarizing the extent of motility observed over a 5 min period are shown in Figure 6 (right panels). As these images and time-lapse recordings clearly illustrate, expression of EGFP-dnCaMKI greatly reduced the overall size of axonal 
growth cones and the size of areas exhibiting motility. Despite these pronounced changed in actin-based motility, axonal transport of phase-dense organelles appeared to be normal (data not shown). It appears that the major effect of dnCaMKI may be on the lamellipodia.

\section{The CaMKK inhibitor STO-609} phenocopies dnCaMKK and dnCaMKI To confirm the effects of dnCaMKI or dnCaMKK using an independent approach, we turned to a selective, cellpermeable CaMKK inhibitor, STO-609 (Tokumitsu et al., 2002, 2003). This inhibitor has an in vitro $\mathrm{IC}_{50}$ of $\sim 0.26 \mu \mathrm{M}(0.1$ $\mu \mathrm{g} / \mathrm{ml}$ ) for CaMKK. A 100 -fold higher concentration $(26 \mu \mathrm{M})$ inhibits CaMKII by only $50 \%$, with essentially no effects on CaMKI, CaMKIV, PKA, PKC, or Erk1. We confirmed the specificity of STO-609 with regard to CaMKK versus CaMKII in cultured hippocampal neurons as follows. Incubation with 2.6 $\mu \mathrm{M}$ STO-609 blocked CaMKK, assessed by its activation of CaMKI and CaMKIV, but it did not inhibit CaMKII (supplemental Fig. S3). STO-609 treatment caused an $\sim 70 \%$ reduction in total neurite length (Fig. 7A), mimicking the effect of dnCaMKK in Figure 2. Importantly, the inhibitory effect of STO-609 was rescued by transfection with a CaMKK mutant $\left(\mathrm{CaMKK}_{\mathrm{L} 233 \mathrm{~F}}\right)$ that has a 10- to 80-fold lower affinity for STO-609 than wild-type CaMKK (Tokumitsu et al., 2003). If the downstream target of CaMKK is CaMKI, constitutively active CaMKI should also reverse the effects of STO-609, and this was observed (Fig. 7A). Moreover, in the absence of STO-609, caCaMKI stimulated axon outgrowth.

We also tested whether STO-609 might inhibit Akt. Akt can be activated through phosphorylation of Ser308 and Ser473 by numerous kinases, including CaMKK (Yano et al., 1998). Under our culture conditions, phosphorylation of Ser308, the site phosphorylated by CaMKK, was barely detectable compared with Ser473; neither was inhibited by STO-609 but both were blocked by the phosphoinositide (PI) 3-kinase inhibitor wortmannin (Fig. S3). We conclude that the low "basal" Akt activation in our neurons is caused by downstream members of the PI 3-kinase pathway (e.g., PDK1) and not by CaMKK. Combined with the fact that dnAkt did not suppress axonal outgrowth (Fig. S2), Akt does not appear to mediate the effects of CaMKK on axonal outgrowth.

Because STO-609 mimicked the effects of dnCaMKK and dnCaMKI on axon elongation, we also examined its effects on growth cone morphology and motility. Incubation with $2.6 \mu \mathrm{M}$ STO-609 caused axonal growth cone collapse that began at $\sim 1 \mathrm{hr}$ and was maximal by $2 \mathrm{hr}$ (Fig. $7 \mathrm{~B}, \mathrm{C}$ ). This time course may be dictated to a large extent by the limited cell permeability of STO-609,
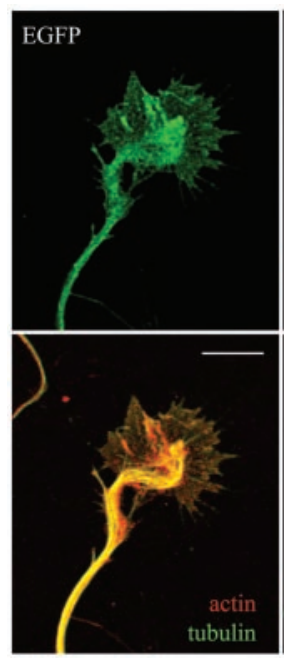

B

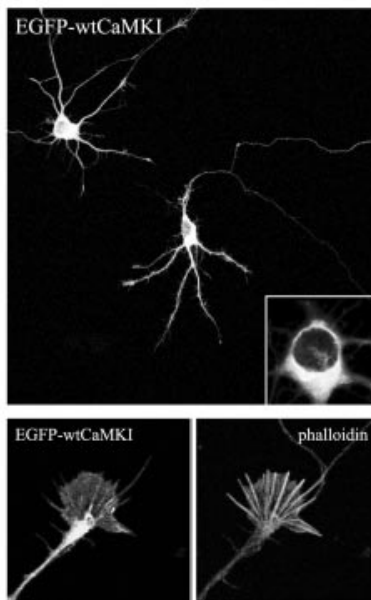

C

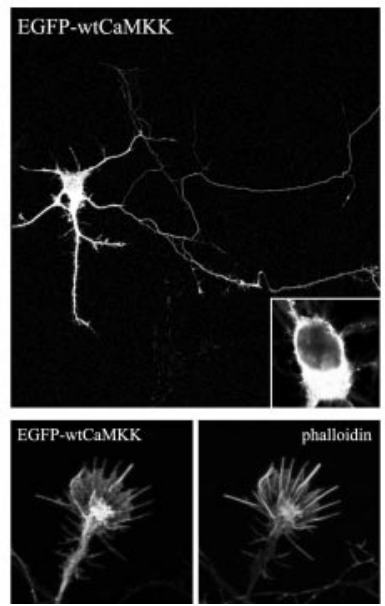

Figure 4. Localization of CaMKI and CaMKK in cultured hippocampal neurons. A, Hippocampal neurons (4 DIV) were immunostained for endogenous CaMKI. B, C, Low-density hippocampal cultures were transfected on day 3 with plasmid encoding EGFP-wtCaMKI ( $B$ ) or EGFP-wtCaMKK ( $C$ ). After $16 \mathrm{hr}$, neurons were fixed and stained with fluorescently labeled phalloidin and imaged by confocal microscopy. Endogenous CaMKI, EGFP-wtCaMKI, and EGFP-wtCaMKK are excluded from the nucleus (insets) but distribute throughout the processes, including the axonal growth cones (bottom panels) extending into the lamellipodia and some, but not all, filopodia. Scale bars: top, $50 \mu \mathrm{m}$; bottom, $10 \mu \mathrm{m}$.
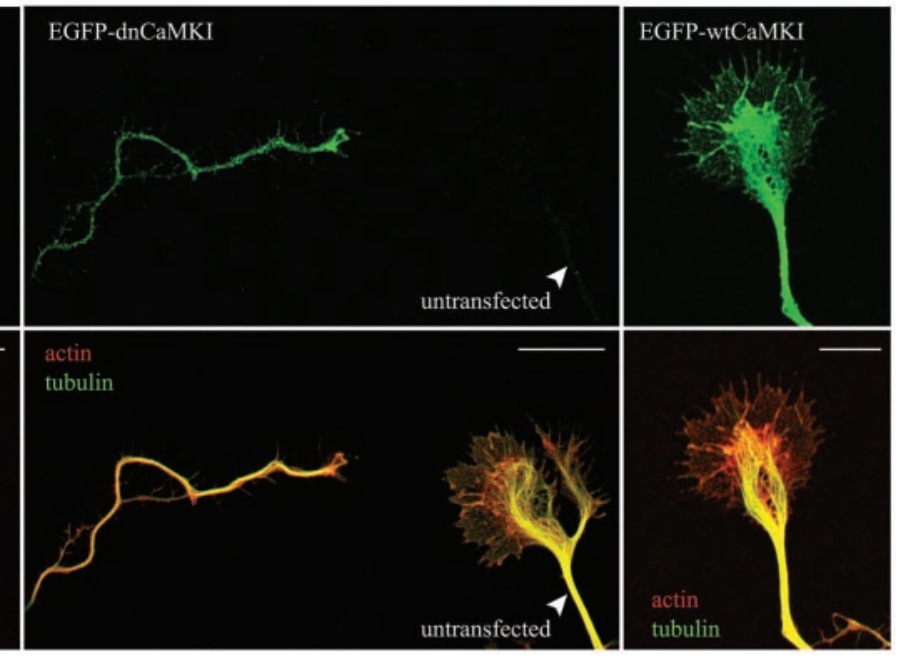

Figure 5. Inhibition of CaMKI results in axonal growth cone collapse. Low-density hippocampal neurons were transfected with constructs encoding dn- and wtCaMKI on day 3 and then fixed $8 \mathrm{hr}$ later. Cells were immunolabeled to stain tyrosinated $\alpha$-tubulin using Cy5-labeled secondary antibodies (pseudocolored green in bottom panel) and stained with tetramethylrhodamine isothiocyanate-labeled phalloidin to visualize filamentous actin (pseudocolored red in bottom panel). In neurons expressing only EGFP (left panel), the peripheral domain of the growth cone is dominated by actin (red), whereas the central domain contains both actin and stabilized microtubules (green; yellow in merged image). Expression of EGFP-dnCaMKI (middle panel, top) dramatically reduced growth cone size (middle panel, left) compared with the growth cone of a neighboring untransfected neuron (middle panel, right). Both peripheral and central domains of the growth cone were essentially eliminated by expression of dnCaMKI. Expression of wtCaMKI had little effect on growth cone morphology (right panel). Scale bar, $10 \mu \mathrm{m}$.

a water-soluble molecule. Treatment of cultured hippocampal neurons with $2.6 \mu \mathrm{M}$ STO-609 also mimicked the effect of dnCaMKI on growth cone motility (supplemental movie 5). Motility of an individual growth cone was recorded for periods of $5 \mathrm{~min}$ at varying times $(5,60$, and $90 \mathrm{~min})$ after STO-609 treatment. Individual frames from such a movie are shown in Figure 7D. After STO-609 treatment for 60 or $90 \mathrm{~min}$, there was a profound decrease in growth cone motility. As with expression of dnCaMKI, STO-609 treatment initially appeared to disrupt lamellipodial structure and inhibit lamellipodial activity, with lesser effects on filopodia. 

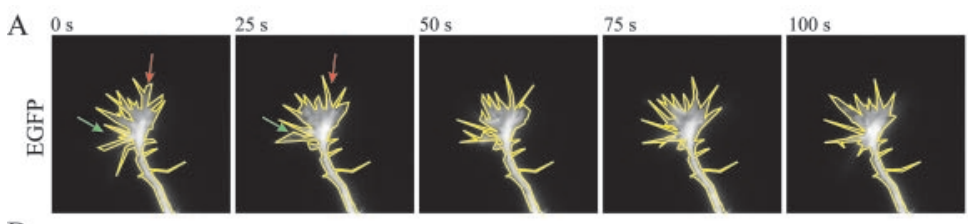

B
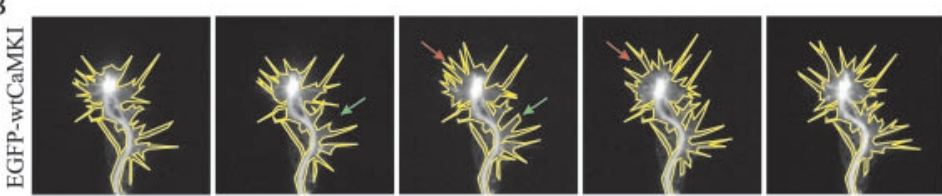

$\mathrm{C}$
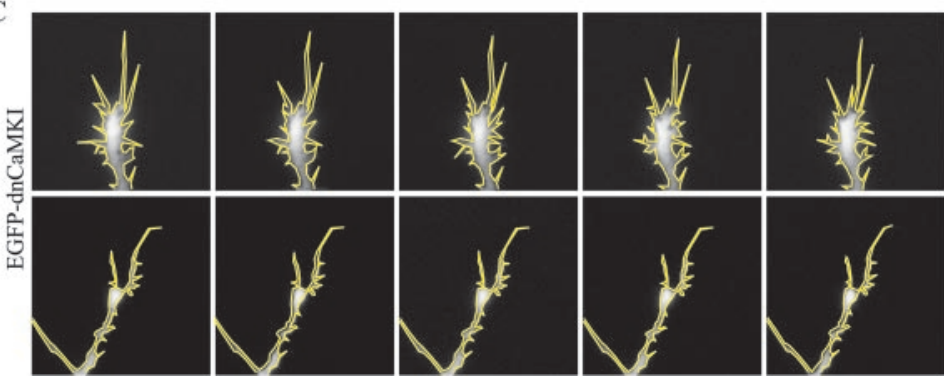

Figure 6. Inhibition of CaMKI suppresses axonal growth cone motility. Axonal growth cone motility was imaged in low-density cultures of 3-d-old hippocampal neurons $8 \mathrm{hr}(A-C$, top panels) or $20 \mathrm{hr}(C$, bottom panels) after transfection with soluble EGFP $(A)$, EGFP-wtCaMKI $(B)$, or EGFP-dnCaMKI ( $C$. Fluorescence images were acquired every 5 sec over a period of 5 min. Individual images taken at $25 \mathrm{sec}$ intervals are shown outlined in yellow. To graphically represent motility (right panel), the area of the growth cone exhibiting motility (occupied by the growth cone in some but not all images) is shown in white. Green arrows highlight areas of growth cone advance; red arrows highlight areas of growth cone retraction. In neurons expressing dnCaMKI, the area explored by a growth cone during 5 min of recording was dramatically reduced compared with the growth cones of control cells expressing EGFP alone or EGFP-wtCaMKI.

\section{Discussion}

The growth cone is a highly specialized structure that responds to multiple extracellular cues to control neurite growth and guidance during development, and calcium mediates some of the major signaling pathways that regulate growth cones. Here we show that the CaMKK-CaMKI pathway is required for normal axon outgrowth and axonal growth cone morphology and motility in cultured hippocampal (stage 4) and cerebellar granule neurons. This conclusion is based on multiple experimental strategies: overexpression of a specific CaMKII inhibitor protein (CaMKIIN), transfection with targeted dominant-negative kinase constructs, and treatment with the CaMKK inhibitor STO609 combined with rescue by an engineered inhibitor-insensitive mutant of CaMKK or by constitutively active CaMKI. These results provide one of the first reports of a physiological role for CaMKI. In addition, our work demonstrates that CaMKII, which was previously thought to mediate stimulatory effects on neurite growth, does not play a major role in early axon extension.

\section{CaMKK signals through CaMKI}

Our results provide very compelling evidence that CaMKK activation of CaMKI mediates axon outgrowth through regulation of its growth cone motility. Dominant-negative constructs of signaling proteins are commonly used to map out signaling pathways (Carpenter and Cantley, 1996). The dnCaMKI and dnCaMKIV likely act by sequestering their upstream activator, CaMKK, rather than their downstream substrate(s). For example, hippocampal slices from transgenic mice expressing dnCaMKIV show a suppressed $\mathrm{Ca}^{2+}$-stimulated CREB phosphorylation without any effect on CREB phosphorylation mediated by elevation of cAMP (Kang et al., 2001). Thus, it is not
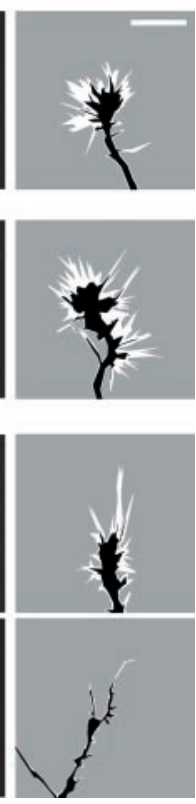

surprising that overexpressed dominantnegative constructs of adjacent members in a signaling cascade (CaMKI and CaMKK) might yield similar effects on neurite development as we observed (Fig. 2 ). We further demonstrate the importance of confirming the correct subcellular distribution of expressed constructs. Our transfected dnCaMKIV was incorrectly localized to the cytoplasm where it suppressed neurite extension, probably by sequestering CaMKK, but when properly confined to the nucleus it had no effect. Finally, we confirmed the results obtained with the dominant-negative CaMKs using a pharmacological approach. The newly characterized CaMKK inhibitor STO-609 (Tokumitsu et al., 2002, 2003) mimicked the effects of dnCaMKK or dnCaMKI on axon extension, growth cone collapse (Fig. 7 ), and growth cone motility (supplemental movie 5) when used at a concentration $(2.6 \mu \mathrm{M})$ that inhibits CaMKK but not CaMKII (supplemental Fig. S3). Most importantly, the inhibitory effect of STO-609 on axon elongation was reversed by expression of the drug-insensitive mutant of CaMKK or constitutively active CaMKI, the downstream target of CaMKK (Fig. 7). These results compel us to conclude that the CaMKK-CaMKI pathway is the in vivo target of STO-609 in axon extension.

The roles of CaMKII and CaMKIV in neurite development Previous studies concerning the role of CaMKII in neurite outgrowth have yielded somewhat conflicting results, some suggesting that CaMKII activation stimulates neurite elongation, and others suggesting that it inhibits elongation (see Introduction). Many of these studies relied on pharmacological manipulations using inhibitors such as $\mathrm{KN}-62$, which subsequent studies have shown blocks several members of the CaMK family. In our experiments, inhibition of CaMKII by expression of CaMKIIN (which does not inhibit CaMKI, CaMKIV, CaMKK, PKA, or PKC) had little or no effect on neurite growth and branching in immature hippocampal and cerebellar granule neurons (Figs. 2, 3). Indeed, it has been reported that activation of CaMKII is relatively refractory in axons, compared with the soma or dendrites, at this stage of development (Menegon et al., 2002), consistent with our conclusion that CaMKII is not important in regulating axonal outgrowth early in development. Because the axon comprises most of the total neurite length in our experiments and the dendrites are immature, our conclusions are only compelling for the developing axon. It appears that CaMKII plays a more important role later in the development of dendrites. For example, Wu and Cline (1998) found that CaMKII inhibits dendritic develop in Xenopus neurons, consistent with a role in stabilizing dendritic arborization, but CaMKII had no effect on the rate of axon outgrowth, axon growth cone dynamics, or the initial formation of dendritic branches. A recent report shows that $\beta$ CaMKII, but not the $\alpha$ isoform, enhances dendritic motility and arborization in hippocampal neurons (Fink et al., 2003). Previous studies had expressed the $\alpha$ isoform, perhaps accounting for much of the previous confusion over the role of CaMKII. In this 
context, it is important to note that CaMKIIN, the inhibitor used in our experiments, effectively blocks both the $\alpha$ and $\beta$ isoforms (Chang et al., 1998).

It has been reported that CaMKIV, probably acting through phosphorylation of the nuclear transcription factor CREB, is a mediator of depolarization-enhanced dendritic growth in cortical neurons (Redmond et al., 2002). Constitutively active CaMKIV enhanced dendritic growth, whereas dnCaMKIV inhibited $\mathrm{KCl}$-stimulated growth; however, the subcellular localization of these CaMKIV constructs was not determined, and overexpressed constitutively active kinases can evoke nonphysiological responses caused by lack of their substrate specificity. In our experiments, dnCaMKIV was expressed predominantly in the cytosol (supplemental Fig. S1A, top panels) where it did inhibit axon extension (Figs. 2, 3), presumably by sequestering CaMKK. Consistent with this mechanism of sequestering CaMKK, dnCaMKI suppresses it own activation by CaMKK (supplemental Fig. S1B); however, when properly targeted to the nucleus with a nuclear localization signal (supplemental Fig. S1 A, bottom panels), dnCaMKIV had no effect on axon extension, although it potently inhibited CREB-dependent gene transcription (Figs. 2, 3). Thus, we conclude that nuclear CaMKIV is not regulatory for axonal outgrowth.

\section{CaMKI and the regulation of growth cone motility}

Transient increases of $\mathrm{Ca}^{2+}$ that are spatially restricted within the growth cone can rapidly alter its morphology and motility, presumably by regulating actin dynamics (Lau et al., 1999; Zheng, 2000; Chadborn et al., 2002; Cheng et al., 2002; Meyer and Feldman, 2002). Our results indicate that CaMKK via its downstream target CaMKI mediates these effects on axonal growth cone motility. CaMKI is present in axonal growth cones, and inhibitors of CaMKI or CaMKK induce growth cone collapse. This inhibition can be overcome by expression of constitutively active CaMKI.

Our current results, combined with recent reports in the literature, extend an emerging theme of signaling specificity mediated by distinct CaMK family members during neuronal development and plasticity. That is, during development, CaMKI mediates axon extension (this report), whereas dendritic arborization is regulated by $\beta$ CaMKII (Fink et al., 2003). In synaptic plasticity, $\alpha$ CaMKII enhances synaptic current by increasing single channel conductance of AMPA receptors (Barria et al., 1997; Derkach et al., 1999; Lee et al., 2000), whereas $\beta$ CaMKII diminishes current (Thiagarajan et al., 2002). Thus, identifying the relevant kinase

D

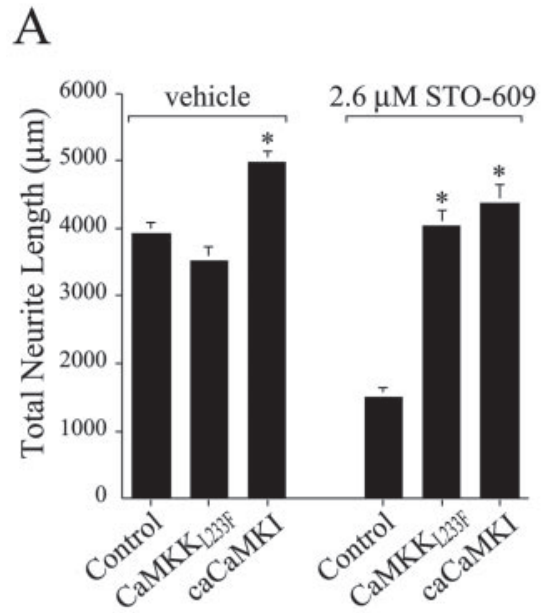

B
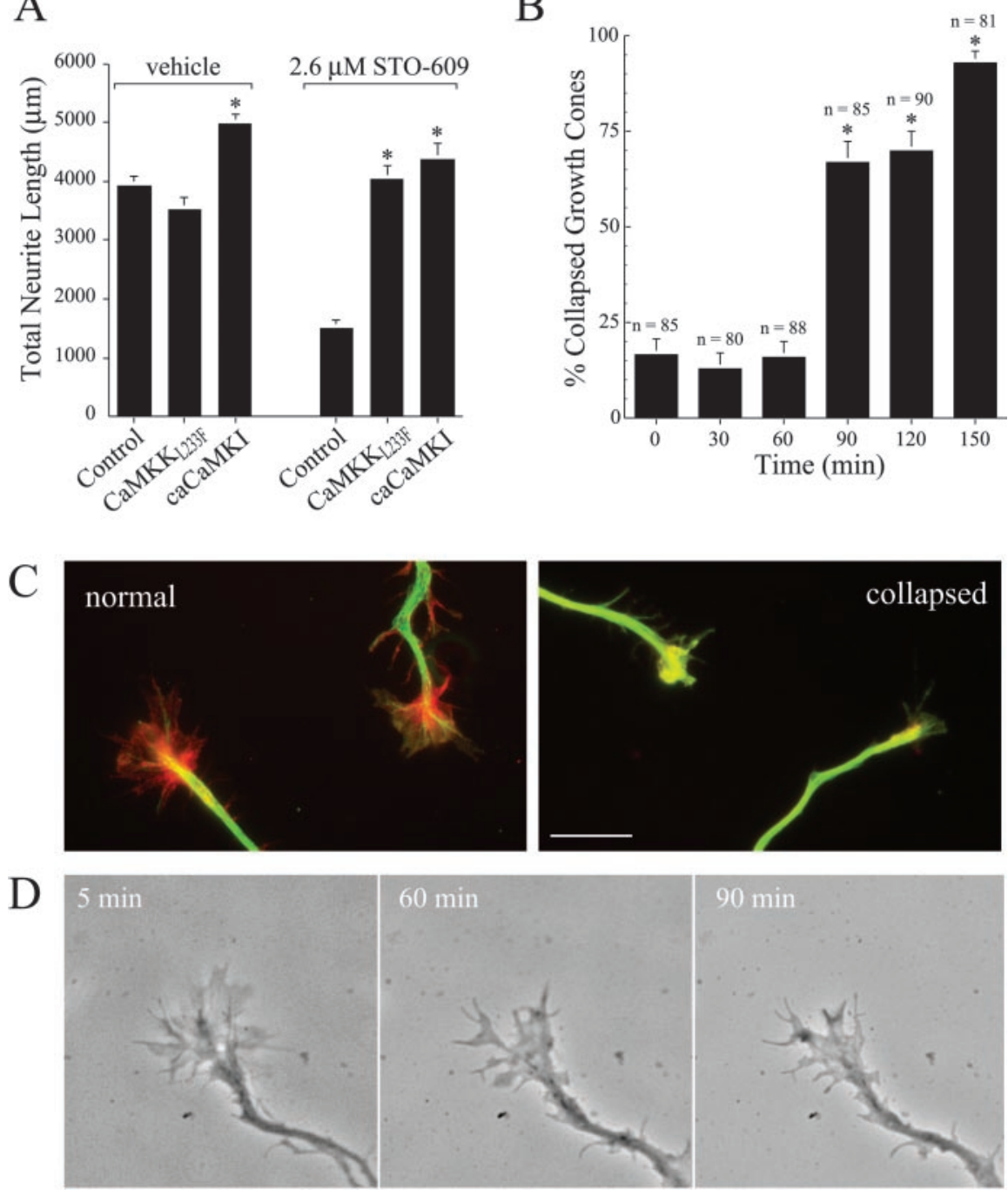

Figure 7. Inhibition of CaMKK by ST0-609 inhibits neurite outgrowth and causes axonal growth cone collapse. A, High-density cultures of rat hippocampal neurons were transfected on day 2 with plasmid encoding soluble EGFP alone (Control) or in combination with ST0-609-insensitive CaMKK $\mathrm{L} 233 \mathrm{~F}_{\mathrm{F}}$ or caCaMKI. Neurons were cultured for $12 \mathrm{hr}$ to allow for expression of the transfected protein and then treated without (i.e., vehicle) or with 2.6 $\mathrm{m}$ ST0-609 for $2 \mathrm{~d}$. Neurons were then fixed and imaged. Quantification of total neurite length is shown for 30 cells per condition. Statistical analysis compares neurite length of kinase-expressing (CaMKK $_{\mathrm{L} 233 \mathrm{~F}}$ or caCaMKI) versus control cells in the absence or presence of ST0-609. * $p$ values of $<0.01$ ( $t$ test). $B, C$, Inhibition of CaMKK by ST0-609 results in growth cone collapse. Low-density cultures of 3-d-old hippocampal neurons were treated with 2.6 $\mu \mathrm{M}$ of ST0-609 and then fixed and stained for filamentous actin (phalloidin; red) and microtubules (green). Randomly selected axonal growth cones (80-100 per condition) were scored as normal ( $C$, left panel) or collapsed ( $C$, right panel) by an investigator without knowledge of the treatment conditions. The bar graph $(B)$ summarizes the percentage of growth cones that were collapsed as a function of time after addition of ST0-609 ( \pm SE of percentage). After 90 min in ST0-609 the percentage of collapsed growth cones quadrupled. Scale bar: $C, 10 \mu \mathrm{m}$. D, Inhibition of CaMKK by ST0-609 suppresses growth cone motility. At 5,60 , or 90 min after addition of $2.6 \mu \mathrm{M}$ ST0-609, axonal growth cone motility was assessed in an individual neuron by acquiring phase-contrast images every $5 \mathrm{sec}$ for a period of $5 \mathrm{~min}$. Single images of these time-lapse recordings show a change from a spread growth cone morphology with lamellar veils extending between filopodia (left) to a reduced growth cone size at $60 \mathrm{~min}$ (middle) and $90 \mathrm{~min}$ (right), with fewer and smaller lamellar extensions. The time-lapse movie available as supplemental material shows that ST0-609 treatment markedly inhibited the motility of this growth cone after 60 and 90 min of treatment. Growth cones of untreated sister cultures showed no reduction of growth cone motility over a period of $120 \mathrm{~min}$ (data not shown).

mediating a physiological response is crucial in that it focuses attention on likely substrates for that kinase. Our study shifts the focus, in terms of axon elongation, away from CaMKII and nuclear CaMKIV and toward CaMKI, particularly its substrates that might regulate growth cone actin dynamics.

There are a large number of phosphoproteins in the growth cone that regulate actin polymerization-depolymerization that 
could be relevant CaMKI targets. Synapsin, the first identified in vitro CaMKI substrate (Nairn and Greengard, 1987), can regulate hippocampal neurite outgrowth in a PKA-dependent phosphorylation pathway (Kao et al., 2002). Because PKA and CaMKI phosphorylate the same site in synapsin I (Nairn and Greengard, 1987), it may be the target for CaMKI. Alternatively, members of the Rho family of GTPases are known to be important regulators of actin filaments in filopodia and lamellipodia (Dickson, 2001; Meyer and Feldman, 2002), and some of the guanine nucleotide exchange factors (GEFs) and GTPase activating proteins (GAPs) that regulate Rho family GTPase activities can be modulated by phosphorylation. Examples include CaMKII-mediated phosphorylation of Ras synaptic GAP (Chen et al., 1998) and the Rac GEF Tiam1 (Fleming et al., 1999) as well as PKA-dependent phosphorylation of the Ras GEF guanine nucleotide releasing factor (Baouz et al., 2001). Because there is redundancy in the substrate specificities for the various CaMKs as well as for PKA, these GEFs and GAPs are potential targets of CaMKI. CaMKI can also phosphorylate myosin II regulatory light chain at the site (Ser19) that stimulates Mg-ATPase activity of myosin II, thereby inducing reorganization of actin filaments (Suizu et al., 2002).

Our identification of CaMKI as a regulatory kinase in $\mathrm{Ca}^{2+}$. mediated neurite outgrowth opens the way to identify the relevant substrates and elucidate the signaling pathways that it initiates. For example, it will be important to determine whether phospho-mimetic constructs of putative CaMKI substrates (e.g., the Ser/Asp mutant of synapsin that mimics its phosphorylation) (Kao et al., 2002) can rescue the inhibitory effects of dnCaMKI or STO-609. Our finding that we can inhibit CaMKK activation of CaMKI in neurons using a pharmacological approach opens the way for the biochemical identification of relevant substrates.

\section{References}

Baouz S, Jacquet E, Accorsi K, Hountondji C, Balestrini M, Zippel R, Sturani E, Parmeggiani A (2001) Sites of phosphorylation by protein kinase A in CDC25Mm/GRF1, a guanine nucleotide exchange factor for Ras. J Biol Chem 276:1742-1749.

Barria A, Muller D, Derkach V, Griffith LC, Soderling TR (1997) Regulatory phosphorylation of AMPA-type glutamate receptors by CaM-KII during long-term potentiation. Science 276:2042-2045.

Brewer GJ (1997) Isolation and culture of adult rat hippocampal neurons. J Neurosci Methods 71:143-155.

Carpenter CL, Cantley LC (1996) Phosphoinositide kinases. Curr Opin Cell Biol 8:153-158.

Chadborn N, Eickholt B, Doherty P, Bolsover S (2002) Direct measurement of local raised subplasmalemmal calcium concentrations in growth cones advancing on an N-cadherin substrate. Eur J Neurosci 15:1891-1898.

Chang BH, Mukherji S, Soderling TR (1998) Characterization of a calmodulin kinase II inhibitor protein in brain. Proc Natl Acad Sci USA 95:10890-10895.

Chang BH, Mukherji S, Soderling TR (2001) Calcium/calmodulindependent protein kinase II inhibitor protein: localization of isoforms in rat brain. Neuroscience 102:767-777.

Chen HJ, Rojas-Soto M, Oguni A, Kennedy MB (1998) A synaptic RasGTPase activating protein (p135 SynGAP) inhibited by CaM kinase II. Neuron 20:895-904.

Cheng S, Geddis MS, Rehder V (2002) Local calcium changes regulate the length of growth cone filopodia. J Neurobiol 50:263-275.

Derkach V, Barria A, Soderling TR (1999) $\mathrm{Ca}^{2+} /$ calmodulin-kinase II enhances channel conductance of alpha-amino-3-hydroxy-5-methyl-4isoxazolepropionate type glutamate receptors. Proc Natl Acad Sci USA 96:3269-3274.

Dickson BJ (2001) Rho GTPases in growth cone guidance. Curr Opin Neurobiol 11:103-110.

Dotti CG, Sullivan CA, Banker GA (1988) The establishment of polarity by hippocampal neurons in culture. J Neurosci 8:1454-1468.

Enslen H, Sun P, Brickey D, Soderling SH, Klamo E, Soderling TR (1994)
Characterization of $\mathrm{Ca}^{2+} /$ calmodulin-dependent protein kinase IV. Role in transcriptional regulation. J Biol Chem 269:15520-15527.

Fink CC, Bayer KU, Myers JW, Ferrell Jr JE, Schulman H, Meyer T (2003) Selective regulation of neurite extension and synapse formation by the beta but not the alpha isoform of CaMKII. Neuron 39:283-297.

Fleming IN, Elliott CM, Buchanan FG, Downes CP, Exton JH (1999) $\mathrm{Ca}^{2+} /$ calmodulin-dependent protein kinase II regulates Tiam1 by reversible protein phosphorylation. J Biol Chem 274:12753-12758.

Gomez TM, Spitzer NC (2000) Regulation of growth cone behavior by calcium: new dynamics to earlier perspectives. J Neurobiol 44:174-183.

Goshima Y, Ohsako S, Yamauchi T (1993) Overexpression of $\mathrm{Ca}^{2+} /$ calmodulin-dependent protein kinase II in Neuro2a and NG108-15 neuroblastoma cell lines promotes neurite outgrowth and growth cone motility. J Neurosci 13:559-567.

Goslin K, Banker G (1998) Rat hippocampal neurons in low-density culture. In: Culturing nerve cells, Ed 2 (Banker G, Goslin K, eds), pp 339370. Cambridge, MA: MIT.

Gringhuis SI, de Leij LF, Wayman GA, Tokumitsu H, Vellenga E (1997) The $\mathrm{Ca}^{2+} /$ calmodulin-dependent kinase type IV is involved in the CD5mediated signaling pathway in human $\mathrm{T}$ lymphocytes. J Biol Chem 272:31809-31820.

Impey S, Fong AL, Wang Y, Cardinaux JR, Fass DM, Obrietan K, Wayman GA, Storm DR, Soderling TR, Goodman RH (2002) Phosphorylation of CBP mediates transcriptional activation by neural activity and CaM kinase IV. Neuron 34:235-244.

Jensen KF, Ohmstede CA, Fisher RS, Sahyoun N (1991) Nuclear and axonal localization of $\mathrm{Ca}^{2+}$ /calmodulin-dependent protein kinase type $\mathrm{Gr}$ in rat cerebellar cortex. Proc Natl Acad Sci USA 88:2850-2853.

Kang H, Sun LD, Atkins CM, Soderling TR, Wilson MA, Tonegawa S (2001) An important role of neural activity-dependent CaM-KIV signaling in the consolidation of long-term memory. Cell 106:771-783.

Kao HT, Song HJ, Porton B, Ming GL, Hoh J, Abraham M, Czernik AJ, Pieribone VA, Poo MM, Greengard P (2002) A protein kinase A-dependent molecular switch in synapsins regulates neurite outgrowth. Nat Neurosci 5:431-437.

Kater SB, Mills LR (1991) Regulation of growth cone behavior by calcium. J Neurosci 11:891-899.

Kuhn TB, Williams CV, Dou P, Kater SB (1998) Laminin directs growth cone navigation via two temporally and functionally distinct calcium signals. J Neurosci 18:184-194.

Lau PM, Zucker RS, Bentley D (1999) Induction of filopodia by direct local elevation of intracellular calcium ion concentration. J Cell Biol 145:1265-1275.

Lee HK, Barbarosie M, Kameyama K, Bear MF, Huganir RL (2000) Regulation of distinct AMPA receptor phosphorylation sites during bidirectional synaptic plasticity. Nature 405:955-959.

Lee JC, Kwon YG, Lawrence DS, Edelman AM (1994) A requirement of hydrophobic and basic amino acid residues for substrate recognition by $\mathrm{Ca}^{2+} /$ calmodulin-dependent protein kinase Ia. Proc Natl Acad Sci USA 91:6413-6417.

Lin CH, Forscher P (1995) Growth cone advance is inversely proportional to retrograde F-actin flow. Neuron 14:763-771.

Lisman J, Schulman H, Cline H (2002) The molecular basis of CaMKII function in synaptic and behavioural memory. Nat Rev Neurosci 3:175-190.

Loseth OP, de Lecea L, Calbet M, Danielson PE, Gautvik V, Hovring PI, Walaas SI, Gautvik KM (2000) Developmental regulation of two isoforms of $\mathrm{Ca}(2+) /$ calmodulin-dependent protein kinase I beta in rat brain. Brain Res 869:137-145.

Matsushita M, Nairn AC (1998) Characterization of the mechanism of regulation of $\mathrm{Ca}^{2+} /$ calmodulin-dependent protein kinase I by calmodulin and by $\mathrm{Ca}^{2+} /$ calmodulin-dependent protein kinase kinase. J Biol Chem 273:21473-21481.

Means AR (2000) Regulatory cascades involving calmodulin-dependent protein kinases. Mol Endocrinol 14:4-13.

Menegon A, Verderio C, Leoni C, Benfenati F, Czernik AJ, Greengard P, Matteoli M, Valtorta F (2002) Spatial and temporal regulation of $\mathrm{Ca}^{2+}$ / calmodulin-dependent protein kinase II activity in developing neurons. J Neurosci 22:7016-7026.

Meyer G, Feldman EL (2002) Signaling mechanisms that regulate actinbased motility processes in the nervous system. J Neurochem 83:490-503. Mochizuki H, Ito T, Hidaka H (1993) Purification and characterization of 
$\mathrm{Ca}^{2+} /$ calmodulin-dependent protein kinase $\mathrm{V}$ from rat cerebrum. J Biol Chem 268:9143-9147.

Nairn AC, Greengard P (1987) Purification and characterization of $\mathrm{Ca}^{2+} /$ calmodulin-dependent protein kinase I from bovine brain. J Biol Chem 262:7273-7281.

Nakamura Y, Okuno S, Kitani T, Otake K, Sato F, Fujisawa H (2001) Immunohistochemical localization of $\mathrm{Ca}(2+) /$ calmodulin-dependent protein kinase kinase beta in the rat central nervous system. Neurosci Res 39:175-188.

Petersen OH, Cancela JM (2000) Attraction or repulsion by local $\mathrm{Ca}(2+)$ signals. Curr Biol 10:R311-314.

Picciotto MR, Cohn JA, Bertuzzi G, Greengard P, Nairn AC (1992) Phosphorylation of the cystic fibrosis transmembrane conductance regulator. J Biol Chem 267:12742-12752.

Picciotto MR, Zoli M, Bertuzzi G, Nairn AC (1995) Immunochemical localization of calcium/calmodulin-dependent protein kinase I. Synapse 20:75-84

Redmond L, Kashani AH, Ghosh A (2002) Calcium regulation of dendritic growth via CaM kinase IV and CREB-mediated transcription. Neuron 34:999-1010.

Rina S, Jusuf AA, Sakagami H, Kikkawa S, Kondo H, Minami Y, Terashima T (2001) Distribution of $\mathrm{Ca}(2+) /$ calmodulin-dependent protein kinase I beta 2 in the central nervous system of the rat. Brain Res 911:1-11.

Sakagami H, Watanabe M, Kondo H (1992) Gene expression of $\mathrm{Ca}^{2+}$ / calmodulin-dependent protein kinase of the cerebellar granule cell type or type IV in the mature and developing rat brain. Brain Res Mol Brain Res 16:20-28.

Sakagami H, Umemiya M, Saito S, Kondo H (2000) Distinct immunohistochemical localization of two isoforms of $\mathrm{Ca}^{2+} /$ calmodulin-dependent protein kinase kinases in the adult rat brain. Eur J Neurosci 12:89-99.

Sawamura Y, Sakagami H, Kondo H (1996) Localization of mRNA for $\mathrm{Ca}^{2+} /$ calmodulin-dependent protein kinase I in the brain of developing and mature rats. Brain Res 706:259-266.

Soderling TR (1999) The Ca-calmodulin-dependent protein kinase cascade. Trends Biochem Sci 24:232-236.

Soderling TR, Chang B, Brickey D (2001) Cellular signaling through multifunctional $\mathrm{Ca}^{2+} /$ calmodulin-dependent protein kinase II. J Biol Chem 276:3719-3722.

Sogawa Y, Yoshimura Y, Otaka A, Yamauchi T (2000) Ca(2+)-independent activity of $\mathrm{Ca}(2+) /$ calmodulin-dependent protein kinase II involved in stimulation of neurite outgrowth in neuroblastoma cells. Brain Res 881:165-175.

Solem M, McMahon T, Messing RO (1995) Depolarization-induced neu- rite outgrowth in PC12 cells requires permissive, low level NGF receptor stimulation and activation of calcium/calmodulin-dependent protein kinase. J Neurosci 15:5966-5975.

Suizu F, Fukuta Y, Ueda K, Iwasaki T, Tokumitsu H, Hosoya H (2002) Characterization of $\mathrm{Ca}^{2+} /$ calmodulin-dependent protein kinase I as a myosin II regulatory light chain kinase in vitro and in vivo. Biochem J 367:335-345.

Takemoto-Kimura S, Terai H, Takamoto M, Ohmae S, Kikumura S, Segi E, Arakawa Y, Furuyashiki T, Narumiya S, Bito H (2003) Molecular cloning and characterization of CLICK-III/CaMKIgamma, a novel membrane-anchored neuronal $\mathrm{Ca}^{2+} /$ calmodulin-dependent protein $\mathrm{ki}$ nase (CaMK). J Biol Chem 278:18597-18605

Tashima K, Yamamoto H, Setoyama C, Ono T, Miyamoto E (1996) Overexpression of $\mathrm{Ca}^{2+} /$ calmodulin-dependent protein kinase II inhibits neurite outgrowth of PC12 cells. J Neurochem 66:57-64.

Thiagarajan TC, Piedras-Renteria ES, Tsien RW (2002) alpha- and betaCaMKII. Inverse regulation by neuronal activity and opposing effects on synaptic strength. Neuron 36:1103-1114.

Tokumitsu H, Chijiwa T, Hagiwara M, Mizutani A, Terasawa M, Hidaka H (1990) KN-62, 1-[ N, O-bis(5-isoquinolinesulfonyl)- $N$-methyl-L-tyrosyl]-4phenylpiper azine, a specific inhibitor of $\mathrm{Ca}^{2+} /$ calmodulin-dependent protein kinase II. J Biol Chem 265:4315-4320.

Tokumitsu H, Inuzuka H, Ishikawa Y, Ikeda M, Saji I, Kobayashi R (2002) STO-609, a specific inhibitor of the $\mathrm{Ca}(2+) /$ calmodulin-dependent protein kinase kinase. J Biol Chem 277:15813-15818.

Tokumitsu H, Inuzuka H, Ishikawa Y, Kobayashi R (2003) A single amino acid difference between alpha and beta $\mathrm{Ca}^{2+} /$ calmodulin-dependent protein kinase kinase dictates sensitivity to the specific inhibitor, STO-609. J Biol Chem 278:10908-10913.

Vaillant AR, Zanassi P, Walsh GS, Aumont A, Alonso A, Miller FD (2002) Signaling mechanisms underlying reversible, activity-dependent dendrite formation. Neuron 34:985-998.

Williams EJ, Mittal B, Walsh FS, Doherty P (1995) A Ca ${ }^{2+} /$ calmodulin kinase inhibitor, KN-62, inhibits neurite outgrowth stimulated by CAMs and FGF. Mol Cell Neurosci 6:69-79.

Wu GY, Cline HT (1998) Stabilization of dendritic arbor structure in vivo by CaMKII. Science 279:222-226.

Yano S, Tokumitsu H, Soderling TR (1998) Calcium promotes cell survival through CaM-K kinase activation of the protein-kinase-B pathway. Nature 396:584-587.

Zheng JQ (2000) Turning of nerve growth cones induced by localized increases in intracellular calcium ions. Nature 403:89-93.

Zheng JQ, Felder M, Connor JA, Poo MM (1994) Turning of nerve growth cones induced by neurotransmitters. Nature 368:140-144. 